SHORT COMMUNICATION

\title{
Construction of a broad host range expression plasmid vector by Golden Gate cloning
}

\author{
Yuh Leng Teo ${ }^{1}$, Wai Keat Toh ${ }^{1}$, Xin Yen Tor ${ }^{1}$, Chai-Ling Ho², Pek Chin Loh ${ }^{1}$ and Hann Ling Wong ${ }^{1 *}$ \\ ${ }^{1}$ Department of Biological Science, Faculty of Science, Universiti Tunku Abdul Rahman, Jalan Universiti, Bandar Barat, \\ 31900 Kampar, Perak Darul Ridzuan, Malaysia. \\ 2Department of Cell and Molecular Biology, Faculty of Biotechnology and Biomolecular Sciences, \\ Universiti Putra Malaysia, Serdang 43400, Selangor Darul Ehsan, Malaysia. \\ Email: hlwong@utar.edu.my
}

Received 27 May 2020; Received in revised form 28 June 2021; Accepted 12 July 2021

\begin{abstract}
Aims: Expression of recombinant proteins across a range of different host organisms has profound contribution to the advancement in biotechnology. In this study, we aimed to construct a highly versatile broad host range (BHR) expression vector, designated as pYL101C.

Methodology and results: The Golden Gate cloning approach was used to construct pYL101C. Key features of pYL101C include a strong integron promoter $\left(P_{I N T C}\right)$, a BHR pBBR1 origin of replication (ori), gentamycin resistance gene $\left(\mathrm{Gm}^{R}\right)$ as a selectable marker and a multiple cloning site (MCS) downstream of the promoter for easy-cloning

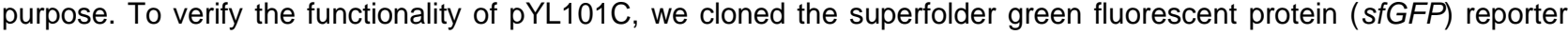
gene into pYL101C and transferred the resultant recombinant plasmid pYL101C::sfGFP into various Gram-negative bacteria. Transformants obtained stably expressed strong green fluorescence under blue light excitation even without selection after four passages.

Conclusion, significance and impact of study: The constructed BHR expression vector, pYL101C and recombinant pYL101C::sfGFP are stable and can be used to monitor the presence of Gram-negative bacteria, such as endophytes and pathogens in their hosts and environment.
\end{abstract}

Keywords: plasmid, expression vector, broad host range, Golden Gate cloning

\section{INTRODUCTION}

Development of versatile plasmid vectors have made significant contributions to the advancements in biotechnology and molecular biology in recent decades (Karsi and Lawrence, 2007; Gawin et al., 2019; de Grahl et al., 2020; Toh et al., 2020). In Gram-negative bacteria, most broad host range vector systems are mainly based on pRSF1010 and pRK2 replicons (Graupner and Wackernagel, 2000; Meyer, 2009). Plasmid stability and the efficiency for transcription and translation of gene-ofinterest are key requisites for an efficient expression vector. Plasmid stability is mainly dependent on the ori, whereas the efficiency of gene expression is determined by the transcriptional control of the promoter within the expression cassette (Rosano and Ceccarelli, 2014). Integron promoters are desirable due to their relatively strong promoter strength and ability to function in a wide range of Gram-negative bacteria (Papagiannitsis et al.,
2009). In this study, we developed a broad host range (BHR) expression vector using Golden Gate cloning approach and designated as PYL101C, consisting of the BHR1 and ColE1 replicons, a strong constitutive integron promoter $\left(P_{I N T C}\right)$ for heterologous gene expression together with a strong transcriptional terminator downstream of the multiple cloning site (MCS) and a gentamycin resistance gene $\left(\mathrm{Gm}^{R}\right)$ selectable marker. Equipped with the pBBR1 ori and $P_{I N T C}$-driven expression cassette, pYL101C can serve multitudinous purposes in molecular biology. We showed application of this vector by stably expressing the superfolder green fluorescent protein (sfGFP) gene in several Gram-negative bacteria.

\section{MATERIALS AND METHODS}

An overview of the construction for plasmids pYL101C and pYL101C::sfGFP is illustrated in Figure 1. Briefly, six DNA fragments were separately amplified by polymerase 
chain reaction (PCR) and subjected to one-pot Golden Gate assembly, utilizing two type IIS restriction enzymes (RE), namely Aarl and Esp3I, and also $T_{4}$ DNA ligase, prior to transformation into competent Escherichia coli cells (Engler et al., 2008). Resultant $\mathrm{Gm}^{\mathrm{R}}$ transformants were screened via colony PCR (Table 1) and positive clones were verified by DNA sequencing. For constructing pYL101C::sfGFP, the sfGFP gene was amplified by PCR (Table 2), cloned into the Notl and Sall sites in the MCS of pYL101C and transformed into competent $E$. coli cells. Recombinant plasmids were extracted from putative transformants and verified by Xbal and Sacl double digestion.

For testing the stability of plasmid pYL101C, E. coli harbouring recombinant pYL101C::sfGFP was streaked on agar plates and incubated overnight at $37{ }^{\circ} \mathrm{C}$. This procedure was repeated for four passages without selection. In each passage, the bacterial colonies were observed for green fluorescence visualization under blue light $(470 \mathrm{~nm})$ excitation. Plasmid pYL101C::sfGFP was subsequently electroporated ( $2500 \mathrm{~V}, 0.2 \mathrm{~cm}$ cuvette) into electro-competent cells of the Gram-negative bacteria, namely Agrobacterium tumefaciens, Klebsiella pneumoniae, Pantoea ananatis and $P$. dispersa as listed in Table 2. All $\mathrm{Gm}^{\mathrm{R}}$ transformants showed strong green fluorescence under blue light $(470 \mathrm{~nm})$ excitation (Figure 2).

\section{RESULTS AND DISCUSSION}

In the Golden Gate cloning approach, all PCR-amplified fragments were flanked with recognition sites of Aarl or Esp3I, which recognize the sequences 5'-CACCTGC-3' and 5'-CGTCTC-3', respectively, and cleave the DNA downstream of their recognition sites. The use of type IIS REs facilitates a scarless cloning as the recognition site would disappear after cloning, leaving no additional RE recognition site in the constructed pYL101C. This cloning approach allows the fast assembly of fragments orderly and simultaneously. The MCS in pYL101C constitutes of several common restriction enzymes recognition sequences, i.e. Notl, Xbal, Sall, Spel and Pmel, allowing facile cloning of gene-of-interest. The MCS is located downstream of PINTC and upstream of T7 terminator, placing the cloned gene(s) under the control of strong

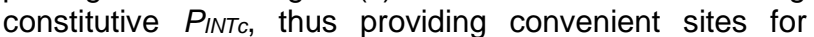
molecular cloning.

Besides, the PINTC would provide high constitutive expression of the gene insert cloned in the MCS. PINTC is one of the strongest promoter combinations and is known to drive the expression of numerous downstream genes. It is functional in a wide range of bacteria (Papagiannitsis et al., 2009). The constitutive expression system of pYL101C permits the activation of transcription and translation of the desired genes without the need of external molecules as inducers and one of the benefits is permitting the growth rate of the cell and the protein synthesis rate to be balanced at intermediate level, saving up the time lost during the lag phase (Geisel, 2011). However, the constitutive expression of gene-of-insert may cause burden to the host cell and affect the expression of desired protein. The presence of lacl gene in PYL101C simplifies the gene expression cassette to be altered into an IPTG-inducible system in the future.

The constructed pYL101C harbours two origins of replication, namely ColE1 replicon and $\mathrm{pBBR} 1$ replicon. The former ori permits high copy per cell and easy genetic manipulation in $E$. coli, whereas the latter allows the vector to be replicated and maintained at medium high copy number in a diverse of Gram-negative bacteria, including Agrobacterium spp. which play pivotal role in plant genetic engineering. The pBBR1 ori in pYL101C
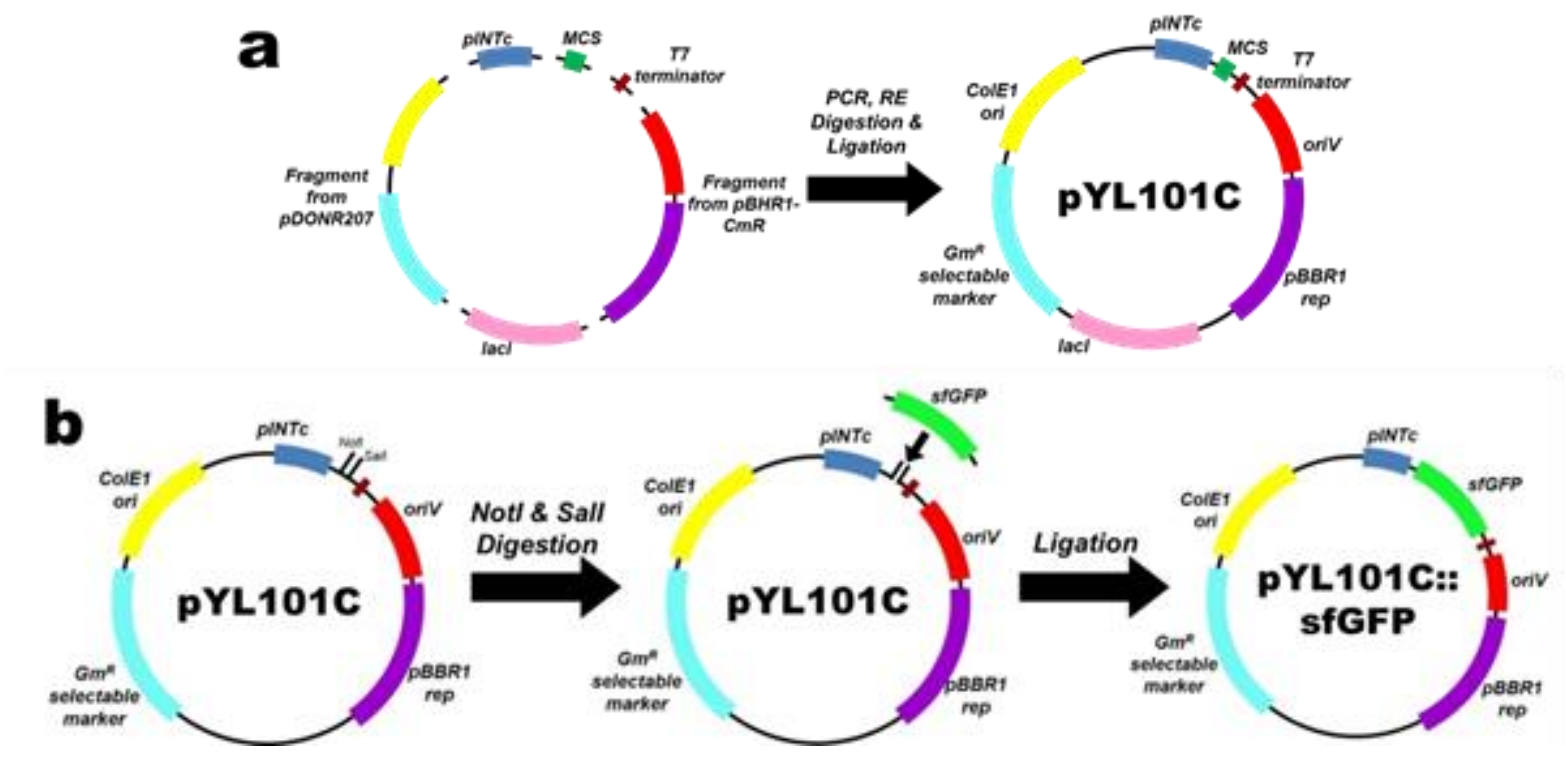

Figure 1: (a) Schematic diagram of the construction of pYL101C (6.0 kb) empty vector. (b) Schematic diagram of the construction of recombinant plasmid, pYL101C::sfGFP (6.7 kb). 
Table 1: List of primers used in this study.

\begin{tabular}{|c|c|c|}
\hline $\begin{array}{l}\text { DNA } \\
\text { fragment }\end{array}$ & Primer & Sequence \\
\hline 1 & F-ColE1 & GTCACCTGCAGTCGAGGTCACTCATGGTGATTTCTCAC \\
\hline 1 & R-rrnbTer & GTCACCTGCTATCGGCTGAGAGTAGGGAACTGCCAG \\
\hline 2 & F-oriV & TGCACCTGCTATTAGGAGCTTGCTCTCCGGGCTTC \\
\hline 2 & R-oriV & TGCACCTGCTGCACGTAGGAACAGCGCACTTACGG \\
\hline 3 & F-pINTc & GTCGTCTCGAGCCGAATTAGACGATATAGGCGCCA \\
\hline 3 & R-pINTc & GTCGTCTCGAAAACTTGTATGTATACTCCTTC \\
\hline 4 & F-T7ter & GTCGTCTCACGCTCACCGCTGAGCAATAACTAG \\
\hline 4 & R-T7ter & GTEGTCTCATCCTCGTCCTGTGGATATCCGGA \\
\hline 5 & F-lacl & GTCGTCTCATACGATCACGCATCTTCCCGACA \\
\hline 5 & R-lacl & GTCGTCTCGCCTCGTTGTAGGTGGACCAGTTG \\
\hline 6 & F-MCS & GGCTACEACCTGCAGTCTTTTGCGGCCGCTCTAGAGTCGACACTAGTGTTTAAAC \\
\hline 6 & R-MCS & GGCTACCACCTGCAGTCAGCGGTTTAAACACTAGTGTCGACTCTAGAGCGGCCGC \\
\hline Colony PCR & F-col-pINTc & TCTTCCGACTGAGCCTTTCG \\
\hline Colony PCR & R-col-oriV & GGCTACGGTCTCAGCGTGCTTTGCGTTCCGTTTGC \\
\hline sfGFP & F-sfGFP & TAGGTCTCAGGCCATGTCAAAAGGAGAAGAGCTG \\
\hline sfGFP & R-sfGFP & TTGTCGACTCTAGAGCGGCCGCTTACTTATAAAGCTCATCCATGCCG \\
\hline
\end{tabular}

${ }^{*}$ The RE sites are underlined and the sticky end generated by RE digestions are in bold letters.

Table 2: List of plasmids and bacteria used in this study.

\begin{tabular}{lll}
\hline Plasmid or bacterial strain & Description & Source \\
\hline pBHR1 & Template for construction of pBHR1-CmR & MoBiTec (2017) \\
pBHR1-CmR & Template for pBHR1 ori & This study \\
pDEST17 & Template for T7 terminator & Invitrogen, USA \\
pDONR207 & Template for ColE1 ori and Gm selectable marker $^{\text {Invitrogen, USA }}$ \\
pING & Template for lacl gene & Toh, unpublished \\
pINT & Template for PINTc promoter & Toh, unpublished \\
pYL101C & BHR expression vector with PINTc promoter & This study \\
pYL101C::sfGFP & Visualization for sfGFP fluorescence & This study \\
E. coli TOP10 & Host for molecular cloning & Invitrogen, USA \\
A. tumefaciens C58 & Host range testing & ATCC 33970 \\
A. tumefaciens LBA4404 & Host range testing & Hoekema et al. (1983) \\
K. pneumoniae & Host range testing & ATCC 13883 \\
$P$. ananatis & Host range testing & Toh et al. (2019) \\
$P$. dispersa & Host range testing & Toh et al. (2019) \\
\hline
\end{tabular}

was derived from pBHR1, a derivative of BHR plasmid pBBR122 (MoBiTec, 2017). Plasmid pBHR1 harbours the $\mathrm{BHR}$ oriV, enabling it to be maintained at medium high copy number in at least 28 Gram-negative microorganisms. This ori is compatible with ColE1 ori as well as commonly used IncP, IncQ and IncW group plasmids. Here, we validated the host range of pYL101C in E. coli and A. tumefaciens and further expand the list of bacterial hosts by including K. pneumonia and Pantoea spp. (Figure 2), which have identified as rice pathogens (Toh et al., 2019). In addition, two A. tumefaciens strains, C58 and LBA4404, which have different chromosomal background, were successfully tested (Deeba et al., 2014). Therefore, pYL101C is expected to be compatible with most Agrobacterium binary vector systems.

The sfGFP reporter gene, which produces bright green fluorescence under blue light excitation, was cloned into pYL101C by RE digestions of Bsal on its 5'-end and
Sall at the 3'-end to produce complementary overhangs to the linearized $p Y L 101 \mathrm{C}$. The recombinant plasmid was verified by visualization of green fluorescence under blue light and subsequently digested with Xbal and Sacl. The expected banding pattern was obtained, indicating the sfGFP insert was successfully cloned. The sfGFP gene was chosen as the reporter gene in this study because it allows non-invasive monitoring and requires no substrate for visualization. The sfGFP protein is well-known for its brightness, improved folding ability and enhanced folding kinetics, even when fused to poorly folded proteins, leading to higher tolerance to chemical denaturants.

The constructed pYL101C::sfGFP enables transformed pathogenic Gram-negative bacteria to emit strong green fluorescence under blue light excitation. Hence, this can be useful for pathogenicity-related study. For instance, pYL101C::sfGFP can be used to visually monitor the infection pathway of Pantoea spp. on rice 


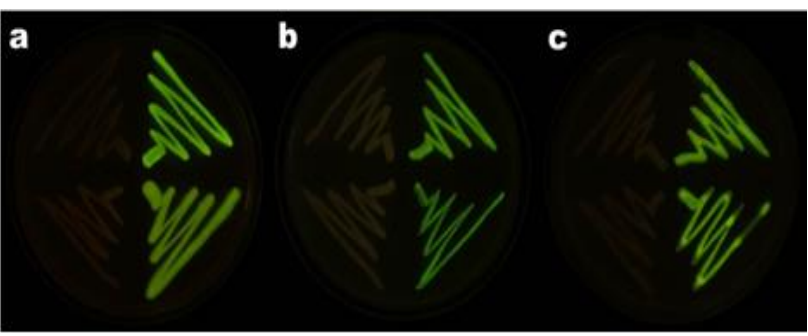

Figure 2: Visualization of sfGFP fluorescence in various Gram-negative bacterial hosts harbouring plasmids, pYL101C empty vector (left) and the recombinant pYL101C::sfGFP (right), under blue light (470 nm) excitation. Both plasmids were tested for plasmid stability in (a) E. coli TOP10 strain (top) and were able to replicate in (a) K. pneumoniae (bottom), (b) A. tumefaciens C58 strain (top), A. tumefaciens LBA4404 strain (bottom), (c) $P$. ananatis (top) and $P$. dispersa (bottom).

plants and thus aids in elucidating its molecular mechanism of pathogenicity and its respective counteractions. The strong constitutive expression of sfGFP reporter protein afforded by the PINTC promoter enables it to provide real-time observation and localization of these bacteria.

In addition, pYL101C could be used as a shuttle vector between $E$. coli and $A$. tumefaciens and utilized in Agrobacterium-mediated transformation (AMT). During AMT, a desired transgene is inserted into a binary vector and co-transformed Agrobacterium cells together with a disarmed $\mathrm{Ti}$ plasmid. The transgene is subsequently transferred and incorporated into host plant chromosome via Agrobacterium type IV secretion system (Wise and Binns, 2016). However, plant transformation efficiency of many crops remained low, thus impeding the application of advanced genome engineering technologies. The virulence genes which are responsible for AMT mostly reside on the Agrobacterium chromosome or the large $\mathrm{Ti}$ plasmid. Low expression of these virulence genes is a bottleneck for efficient plant transformation. The 6-kb pYL101C with medium copy number may boost virulence genes expression, thus improving plant transformation efficiency.

\section{CONCLUSION}

In a nutshell, we showed the ease of using Golden Gate cloning approach in constructing the plasmid vector pYL101C which is a BHR expression vector that can be used in a diverse of Gram-negative bacteria. The strong sfGFP expression from the recombinant pYL101C::sfGFP reflected the functionality of the expression system in these bacterial strains, including $A$. tumefaciens, which is widely used for plant transformation. This plasmid vector may be used in research that involves manipulation of the genetics of Gram-negative bacteria, such as Agrobacterium, for improving plant transformation and elucidation of molecular mechanism of pathogenicity of Pantoea spp.

\section{ACKNOWLEDGEMENTS}

This research was supported by Ministry of Higher Education Malaysia (MOHE) and Universiti Tunku Abdul Rahman through the Fundamental Research Grant Scheme (FRGS) of project no. FRGS/1/2018/STG05/UTAR/02/2 and UTARRF project no. IPSR/RMC/UTARRF/2017-C2/W02, respectively.

\section{REFERENCES}

de Grahl, I., Rout, S. S., Maple-Grødem, J. and Reumann, S. (2020). Development of a constitutive and an auto-inducible high-yield expression system for recombinant protein production in the microalga Nannochloropsis oceanica. Applied Microbiology and Biotechnology 104, 8747-8760.

Deeba, F., Hyder, M. Z., Shah, S. H. and Naqvi, S. M. S. (2014). Multiplex PCR assay for identification of commonly used disarmed Agrobacterium tumefaciens strains. SpringerPlus 3, 358.

Engler, C. Kandzia, R. and Marillonnet, S. (2008). A one pot, one step, precision cloning method with high throughput capability. PLOS ONE 3(11), e3647.

Gawin, A., Peebo, K., Hans, S., Ertesvåg, H., Irla, M., Neubauer, P. and Brautaset, T. (2019). Construction and characterization of broad-host-range reporter plasmid suitable of on-line analysis of bacterial host responses related to recombinant protein production. Microbial Cell Factories 18, 80.

Geisel, N. (2011). Constitutive versus responsive gene expression strategies for growth in changing environments. PLOS ONE 6(11), e27033.

Graupner, S. and Wackernagel, W. (2000). A broadhost-range expression vector series including a Ptac test plasmid and its application in the expression of the dod gene of Serratia marcescens (coding for riboluse-5-phosphate 3-epimerase) in Pseudomonas stutzeri. Biomolecular Engineering 17(1), 11-16.

Hoekema, A., Hirsch, P. R., Hooykaas, P. J. J. and Schilperoort, R. A. (1983). A binary plant vector strategy based on separation of vir- and T-region of the Agrobacterium tumefaciens Ti-plasmid. Nature 303, 179-180.

Karsi, A. and Lawrence, M. L. (2007). Broad host range fluorescence and bioluminescence expression vectors for Gram-negative bacteria. Plasmid 57(3), 286-295.

Meyer, R. (2009). Replication and conjugative mobilization of broad host-range IncQ plasmids. Plasmid 62(2), 57-70.

MoBiTec (2017). Broad host range vectors pBBR122 and pBHR1. MoBiTec GmbH, Germany.

Papagiannitsis, C. C., Tzouvelekis, L. S. and Miriagou, V. (2009). Relative strengths of the class 1 integron promoter hybrid 2 and the combinations of strong and hybrid 1 with an active P2 promoter. Antimicrobial Agents and Chemotherapy 53(1), 277-280.

Rosano, G. L. and Ceccarelli, E. A. (2014). Recombinant protein expression in Escherichia coli: 
Malays. J. Microbiol. Vol 17(5) 2021, pp. 588-592

DOI: http://dx.doi.org/10.21161/mjm.211184

Advances and challenges. Frontiers in Microbiology 5, 172.

Toh, W. K., Loh, P. C. and Wong, H. L. (2019). First report of leaf blight of rice caused by Pantoea ananatis and Pantoea dispersa in Malaysia. Plant Disease 103(7), 1764.

Toh, W. K., Loo, E. P., Tee, C. S., Loh, P. C. and Wong, H. L. (2020). Development of acetosyringoneinducible Gateway ${ }^{\circledR}$ and Golden Gate expression vectors for heterologous gene expression in Agrobacterium tumefaciens. In Vitro Cellular and Developmental Biology - Plant 56(5), 578-587.

Wise, A. A. and Binns, A. N. (2016). The receiver of the Agrobacterium tumefaciens VirA histidine kinase forms a stable interaction with VirG to activate virulence gene expression. Frontiers in Microbiology 6, 1546. 